\title{
Predicting infusion pressure during pars plana vitrectomy: a physically based model
}

Tommaso Rossi ${ }^{1}$, Giorgio Querzoli ${ }^{2}$, Giampiero Angelini ${ }^{3}$, Alessandro Rossi ${ }^{3}$, Carlo Malvasi $^{3}$, Laura Landi ${ }^{1}$, Serena Telani ${ }^{1}$, Guido Ripandelli ${ }^{4}$

${ }^{1}$ Scientific Institute for Research and Healthcare Polyclinical San Martino, Genoa, Italy; ${ }^{2}$ University of Cagliari, DICAAR, Cagliari, Italy; ${ }^{3}$ Optikon 2000 Inc., Rome, Italy; ${ }^{4}$ Scientific Institute for Research and Healthcare G.B. Bietti Foundation, Rome, Italy

\section{Abstract}

Purpose: Intraocular pressure (IOP) during pars plana vitrectomy (PPV) decreases as aspiration generates flow, a phenomenon known as head loss. Since direct measurement of the IOP during surgery is impractical, currently, available compensating systems infer IOP by measuring infusion flow rate and estimating corresponding pressure drop. The purpose of the present paper is to propose and validate a physically based algorithm of the infusion pressure drop as a function of flow.

Methods: Complete infusion lines (20G, 23G, 25G and 27G) were set up and primed. The infusion bottle was set at incremental heights and flow rate measured 10 times and recorded as mean \pm SD. Overall head loss $(\mathrm{OHL})$ was defined, according to hydraulics laws, as the sum of frictional head loss (FHL; i.e., pressure drop due to friction along tubing) and exit head loss (EHL). The latter is equal to the kinetic energy of the exiting flow through the trocar $\left(F K E=V^{2} / 2 \mathrm{~g}\right)$. A $2^{\text {nd }}$ degree polynomial equation (i.e., $\Delta \mathrm{P}=\mathrm{aQ}^{2}+\mathrm{bQ}$, where $\Delta \mathrm{P}$ is the pressure drop, or $\mathrm{OHL}$, and $\mathrm{Q}$ is the volumetric flow) was derived for each gauge and compared to experimental data $2^{\text {nd }}$ order polynomial best-fit curve.

Results: Ninety-seven percent of the pressure values for all gauges predicted using the derived equation fell within 2 SD of the mean difference yielding a Bland-Altman statistical significance when compared to $91 \%$ of best fit curve.

Conclusion: The derived equations accurately predicted the head loss for each given infusion line gauge and can help infer IOP during PPV.

Correspondence: Tommaso Rossi MD, Via Benedetto XV 5, 16132 Genoa, Italy.

E-mail: tommaso.rossi@usa.net 
Keywords: head loss, intraocular pressure compensation, pars plana vitrectomy, volumetric flow rate

\section{Introduction}

Pars plana vitrectomy (PPV) addresses a variety of indications, including retinal detachment, diabetic retinopathy, macular surgery, and many others. Maintaining invariant intraocular pressure (IOP) and therefore volume during surgery, minimizes bleeding and prevents serious untoward events, as listed by Rossi et al. ${ }^{1}$, including hemorrhagic choroidal detachment, optic nerve ischemia, and vitreous hemorrhage.

Infusion bottle height guarantees stable intraocular volume and equals ocular pressure under steady conditions (i.e., when there is no flow). As the aspiration starts, balanced salt solution (BSS) (Alcon, Fort Worth, USA) flows through the infusion line and dissipates part of its energy, thus reducing pressure, a phenomenon called head loss. In order to maintain a stable IOP during aspiration, additional infusion pressure is needed to compensate the effect of head loss. ${ }^{2}$ Conversely, when aspiration stops, flows also reduces to zero: pressure drop instantaneously vanishes and IOP suddenly increases. ${ }^{3}$

Several systems intended to compensate head loss have been developed and their efficiency has been tested by Falabella ${ }^{4}$ and Okamoto, ${ }^{5}$ although the underlying physics and algorithms used often remain unknown.

The purpose of the present paper is to characterize the pressure drop due to head loss of different infusion tubes ranging in diameter from 20G to $27 \mathrm{G}$ (Table 1) as a function of volumetric flow rate, in order to develop a physically based algorithm capable of calculating infusion pressure drop. This model applies to any given infusion system and allows the prediction of head loss based on the flow measurement and the deployment of reliable compensating systems. A thorough comprehension of head loss will also help design more efficient infusion lines.

Table 1. Gauge and corresponding inner diameter (in $\mathrm{mm}$ ).

\begin{tabular}{|l|l|}
\hline Gauge & Infusion cannula inner diameter \\
\hline $20 \mathrm{G}$ & 0.69 \\
\hline $23 \mathrm{G}$ & 0.52 \\
\hline $25 \mathrm{G}$ & 0.40 \\
\hline $27 \mathrm{G}$ & $0.43^{\star}$ \\
\hline
\end{tabular}

"Note that "inner diameter" is considered the actual free surface available to fluid flow. The material we tested adopted the solution of an infusion cannula within the trocar for 20G, $23 \mathrm{G}$, and $25 \mathrm{G}$, while for the $27 \mathrm{G}$ infusion there was no cannula as the infusion tubing was directly connected to the trocar itself, which acted as an infusion cannula without further lumen reduction in order to increase flow. This is why the $27 \mathrm{G}$ inner lumen is actually wider than the 25G. 


\section{Materials and methods}

\subsection{Experimental setting}

A sketch of the experimental setup is shown in Figure 1. The infusion system full of BSS, disposable tubing, cassette, 3-way stopcock, and four infusion cannulas of different gauges, 20G, 23G, 25G, and 27G (Table 1), were set up in the usual fashion and primed. All tests used the R-Evolution CR800 combined phaco-vitrectomy machine (Optikon 2000 Inc., Rome, Italy). In order to exclude possible vacuum within the infusion bottle, a plastic container open at the top was used and BSS continuously refilled to maintain the free surface height constant. The infusion ran by gravity and the cannula was taped to a plastic graduated beaker so that fluid discharge was horizontal at a given height and air-free.

\subsection{Volumetric flow measures}

The infusion bottle was set at incremental heights (measured from the discharge level) and the resulting volumetric flow recorded. During each experiment, the flow rate was measured by gathering fluid within the graduated container (Fig. 1, no.5) for 60 seconds after discarding the first 5 seconds of transient flow. The same procedure was repeated 10 times for each given height, $\mathrm{z}_{1}$, and gauge and flow recorded as mean \pm standard deviation (Fig. 2).

\subsection{Physical-mathematical model and algorithm}

The setting of the vitrectomy infusion system during surgery is represented in Figure 3.

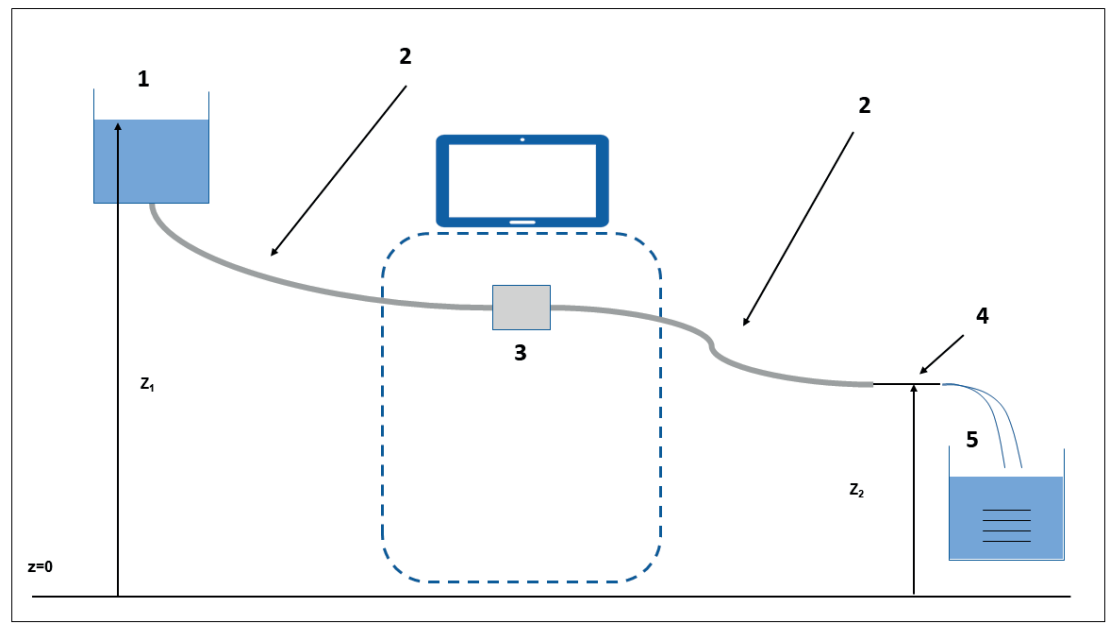

Fig. 1. Sketch of the setup used during the experiments: (1) Open bottle; (2) tubing; (3) vitrectomy machine and cassette; (4) cannula; (5) graduated beaker. 


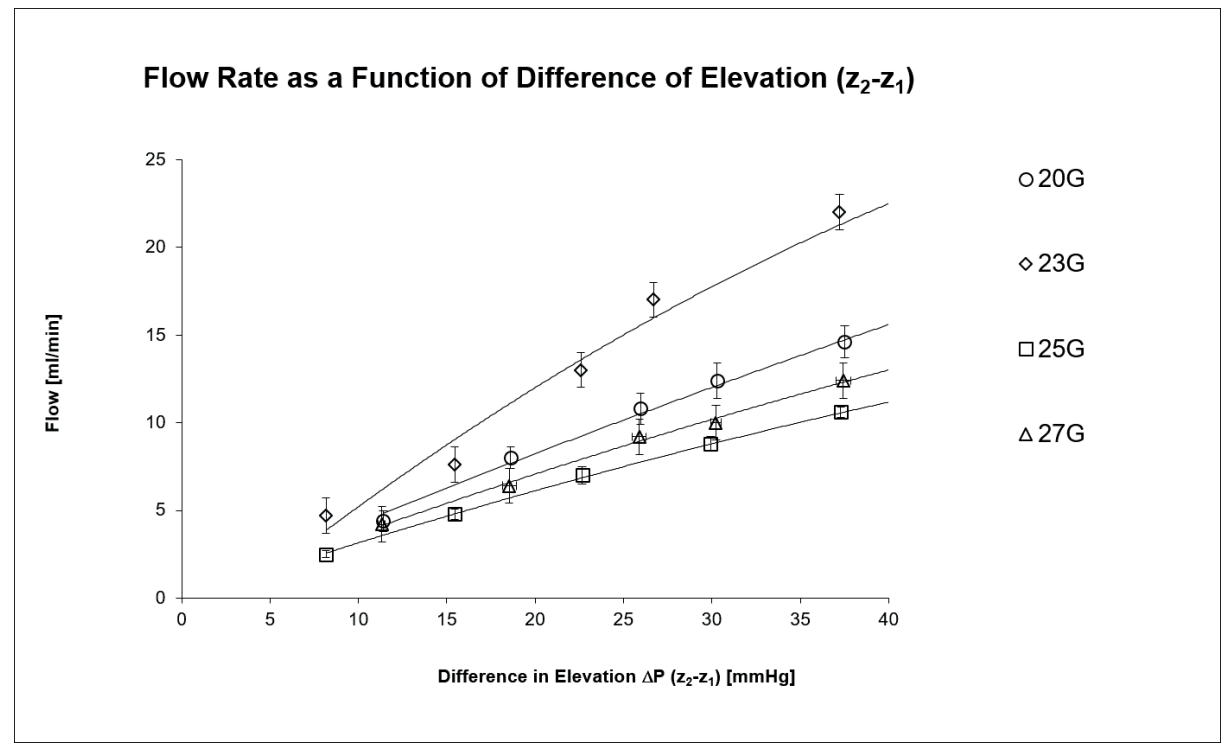

Fig. 2. Infusion line $\mathrm{OHL}$ as a function of flow.

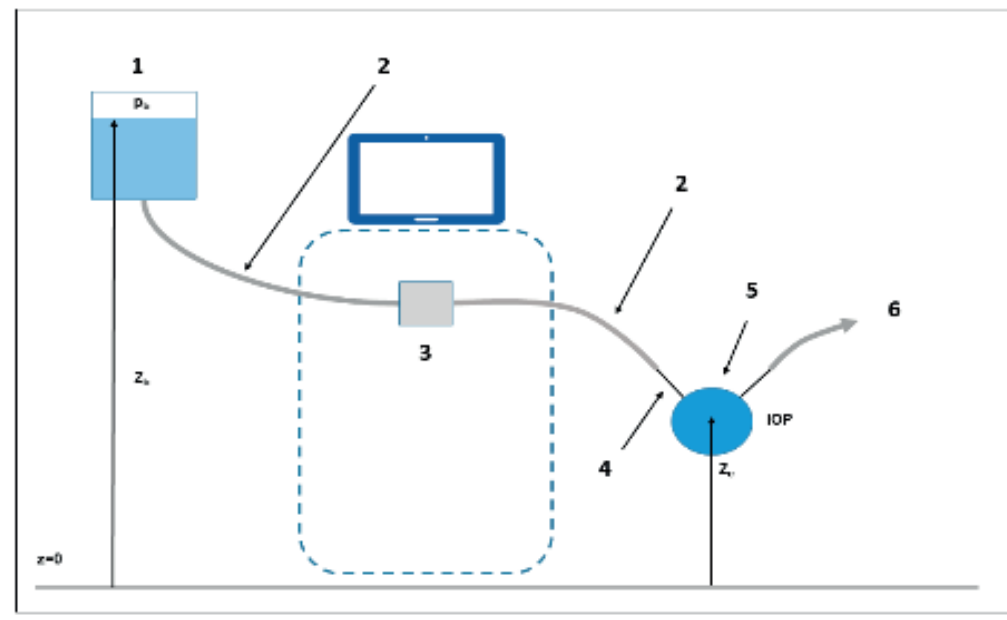

Fig. 3. Sketch of the system setup during surgery. (1) Irrigation bottle; (2) cannula; (3) vitrectomy machine with disposable cassette; (4) irrigation tubing; (5) eye; (6) aspiration. 
In general, the mechanical energy per unit weight of the fluid (the so-called total head, $\mathrm{H}$ ) is given by: $\mathrm{H}=\mathrm{z}+\mathrm{p} / \mathrm{\gamma}+\mathrm{v} 2 /(2 \mathrm{~g})$, where $\mathrm{z}$ is the elevation, $\mathrm{p}$ the relative pressure (i.e., referred to the atmospheric pressure), $\gamma$ the specific weight of the fluid, $v$ the fluid velocity, and $g$ the gravitational acceleration. The total head at the BSS bottle (Fig. 3, no. 1) is:

$$
\mathrm{H}_{\mathrm{b}}=\mathrm{Z}_{\mathrm{b}}+\mathrm{p}_{\mathrm{b}} / \mathrm{v}
$$

under the assumption that the average velocity in the bottle is negligible. pb indicates the venting pressure if present (i.e., when the pressure is equalized to ambient pressure through the opening of an electromagnetic valve $p_{b}=0$; conversely, if the bottle is pressurized, $\mathrm{pb}$ indicates the pressure within the bottle) and $\mathrm{z}_{\mathrm{b}}$ is the elevation of the free surface. Similarly, the total head in the eye is given by:

$$
H_{e}=z_{e}+10 P / \%
$$

where $z_{e}$ is the elevation of the eye and it is assumed that the average velocity of the fluid within the eye is negligible. When the fluid is at rest, the total head in the bottle and in the eye are the same $\left(\mathrm{H}_{\mathrm{e}}{ }_{\mathrm{e}}=\mathrm{H}_{\mathrm{b}}\right)$. Equating the heads and rearranging the terms yields:

$$
I O P^{\prime}=\gamma\left(Z_{b}-Z_{e}\right)+P_{b}
$$

As the flow from the bottle to the eye starts, a portion OHL of the fluid energy is dissipated along the infusion system and the total head (mechanical energy) in the eye, $\mathrm{H} " \mathrm{e}=\mathrm{ze}+\mathrm{IOP} " / \mathrm{Y}$ decreases correspondingly:

$$
\mathrm{H} " \mathrm{e}=\mathrm{H}_{\mathrm{b}}-\mathrm{OHL}
$$

Substituting the expression of H'e and Equation (2) in Equation (4), IOP during infusion results in:

$$
I O P^{\prime \prime}=\gamma\left(Z_{b}-Z_{e}\right)+P_{b}-\gamma O H L=I O P^{\prime}-\gamma O H L
$$

Equation (4) states that the pressure drop given by the infusion flow, PD = IOP' - IOP", is proportional to the $\mathrm{OHL}$ along the infusion: $\mathrm{PD}=\gamma \mathrm{OHL}$. $\mathrm{OHL}$ may be ideally divided into two contributions: the frictional dissipation of energy (per unit weight) along the infusion tubing (frictional head loss; $\mathrm{FHL}$ ) and the dissipation of energy (per unit weight) at the cannula outlet (exit head loss; EHL):

$$
\mathrm{OHL}=\mathrm{FHL}+\mathrm{EHL}
$$


We measured the FHL experimentally by means of the setup described in Figure 1 and computed the EHL analytically.

In order to measure the FHL during the experiments, the total head was measured both at the beginning (the bottle in Fig. 1, no.1; $\mathrm{H}_{1}$ ) and at the end of the infusion line (the cannula in Fig. 1, no. 4; $\left.\mathrm{H}_{2}\right)$. The difference $\left(\mathrm{H}_{1}-\mathrm{H}_{2}\right)$ yields the head loss due to frictional energy dissipation along the infusion tubing FHL.

The total head at the (open) bottle level corresponds to the free surface elevation $\left(H_{1}=z_{1}\right)$, since both relative pressure and velocity vanish at the open bottle surface, whereas the total head at the exit, $\mathrm{H}_{2}$, is the sum of elevation $\left(\mathrm{z}_{2}\right)$ and final kinetic energy (per unit weight), $\mathrm{FKE}=\mathrm{v}_{2}{ }^{2} / 2 \mathrm{~g}$, since the outlet is in air and the relative pressure is null:

$$
\mathrm{H}_{2}=\mathrm{z}_{2}+\mathrm{v}_{2}^{2} / 2 \mathrm{~g}=\mathrm{z}_{2}+\mathrm{FKE}
$$

where $\mathrm{v}_{2}$ indicates the fluid velocity at the outlet. Fluid velocity $\mathrm{v}_{2}$ can be calculated as the volumetric flow rate (FR) divided by the area of the exit section (the infusion cannula) (A): $v_{2}=F R / A$.

As mentioned above, $\mathrm{FHL}$ is the difference between the total heads at the ends of the tubing:

$$
\mathrm{FHL}=\mathrm{H}_{2}-\mathrm{H}_{1}=\left(\mathrm{z}_{2}-\mathrm{z}_{1}\right)-\mathrm{FKE}
$$

i.e., the frictional dissipation along the tubing. The FHL can be obtained from the experiments simply by subtracting the FKE from the difference of elevation $\left(\mathrm{DE}=\mathrm{z}_{1}-\mathrm{z}_{2}\right)$.

The second contribution to $\mathrm{OHL}$, i.e., EHL, can be evaluated by considering that, during surgery, the cannula is inserted into the posterior chamber of the eye (Fig. 3, no. 2), and once within the eye, the fluid moves erratically until it dissipates all the kinetic energy it had at its entrance through the infusion cannula (i.e., the FKE). Therefore, EHL is exactly equal to FKE. In the present experiments, we calculated this additional head loss as:

$$
E H L=F R^{2} /\left(2 g A^{2}\right)
$$

Combining Equation (6) with Equations (8) and (9), we obtain that OHL in surgical conditions is directly proportional to the difference in elevation $\left(D E=z_{1}-z_{2}\right)$ imposed during our experiments. $\triangle \mathrm{p}=\mathrm{\gamma OHL}$ is the pressure drop that must be compensated in order to maintain a constant IOP during surgical maneuvers irrespective of the infusion rate. Following the above argument, the pressure drop can be considered the sum of the contribution of the FHL, $\Delta \mathrm{p}_{\mathrm{FHL}}=\gamma \mathrm{FHL}$ and $\mathrm{EHL}, \Delta \mathrm{p}_{\mathrm{EHL}}=\gamma E H L$.

During our measurements, we imposed the DE and measured the FR for each given experiment and analytically computed the EHL from Equation (9). Then, we 
derived the FHL as the difference between DE and FKE (Equation (8)).

The resulting values are reported in the left panes of Figures 4-7 for 20 G, 23G, 25G, and $27 \mathrm{G}$ infusion tubing, respectively. In the same plots, the values of EHL obtained on the basis of Equation (9) are reported.

Obtained FHL values are fairly aligned along a straight line, demonstrating a linear dependency of the head loss on the flow rate, as expected in laminar flows. ${ }^{6}$ This result is consistent with the fact that the Reynolds number, $R e=U D / v$ ( $U$ indicates the velocity of the flow, $D$ the inner diameter of the infusion conduit, and $v$ the kinematic viscosity of the fluid) in the infusion line did not exceed $R e=400$ during our tests, well below the value of transition to turbulence in pipe flows. ${ }^{7}$ FHL for the different configurations has been approximated as a function of the flow rate by a linear best fit of experimental data points (see Equation (5) in Appendix), as reported in Figures 4a-7a. These two equations (parabolic Equation (9) and linear best fit), calculated for each given gauge, can be algebraically added to yield a third function (referred to as the 'derived' equation or function from here on) that can be used to predict the $\mathrm{OHL}$ along the infusion line for any given flow rate (see Appendix).

Finally, the values of $\mathrm{OHL}$ predicted by the above-mentioned function were compared to experimental data to verify whether they matched (Fig. 8).

Both observed and calculated OHL values have been plotted in Figure 9a-9d for 20G, 23G, 25G, and 27G, respectively. Intuitively, the lesser the distance between observed and calculated data points, the better the predictive capability of the algorithm.

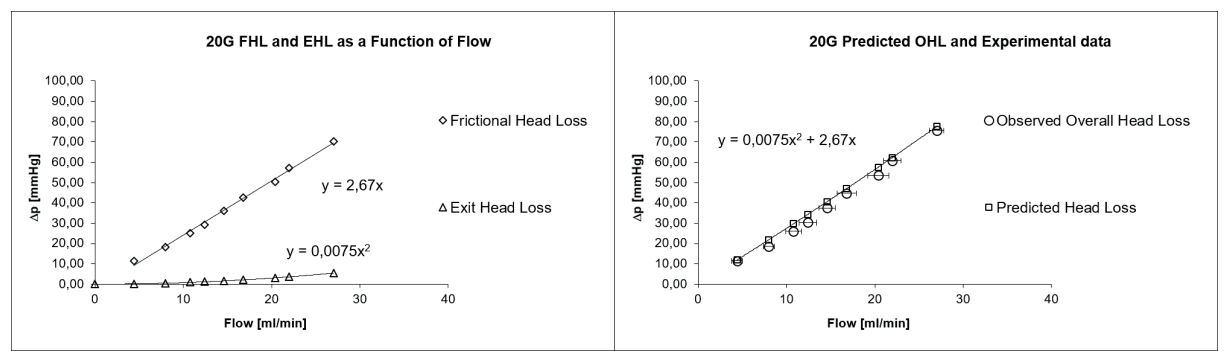

Fig. 4. $20 \mathrm{G}$ infusion. (Left) $\mathrm{EHL}$ and $\mathrm{FHL}$ as functions of observed volumetric flow. Both curves and derived equations (EHL, parabolic and FHL, linear) are reported in each graph. (Right) Measured OHL compared to predictions by the derived equation (reported in the plot). Experimental data points (observed OHL) and calculated values (predicted head loss) overlap significantly. 


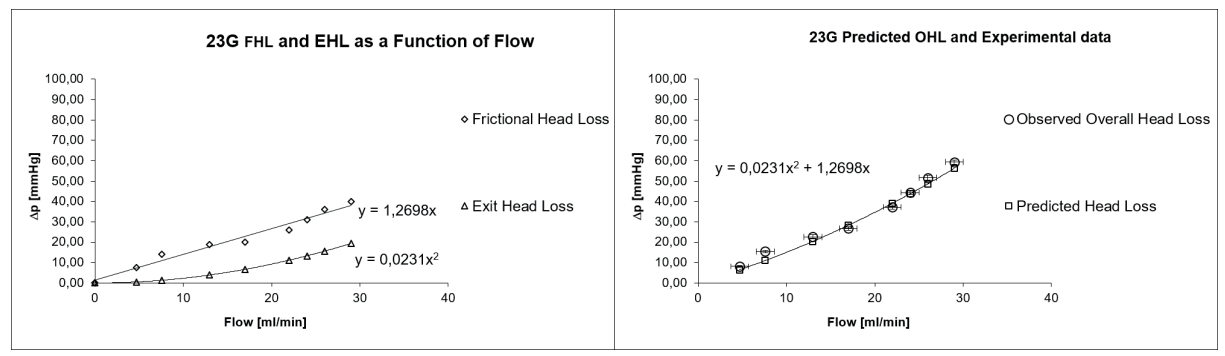

Fig. 5. $23 \mathrm{G}$ infusion. (Left) EHL and FHL as functions of observed volumetric flow. Both curves and derived equations (EHL, parabolic and FHL, linear) are reported in each graph. (Right) Measured OHL compared to predictions by the derived equation (reported in the plot). Experimental data points (observed $\mathrm{OHL}$ ) and calculated values (predicted head loss) overlap significantly.

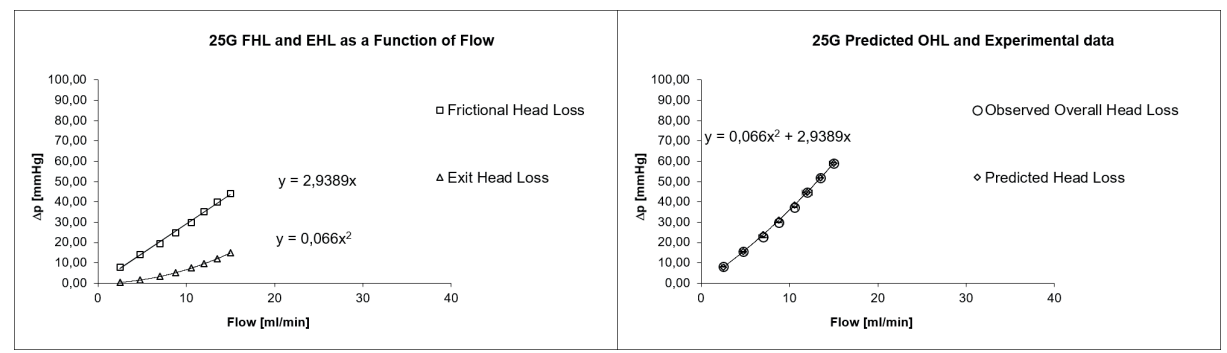

Fig. 6. $25 \mathrm{G}$ infusion. (Left) $\mathrm{EHL}$ and FHL as functions of observed volumetric flow. Both curves and derived equations (EHL, parabolic and FHL, linear) are reported in each graph. (Right) Measured OHL compared to predictions by the derived equation (reported in the plot). Experimental data points (observed $\mathrm{OHL}$ ) and calculated values (predicted head loss) overlap significantly.

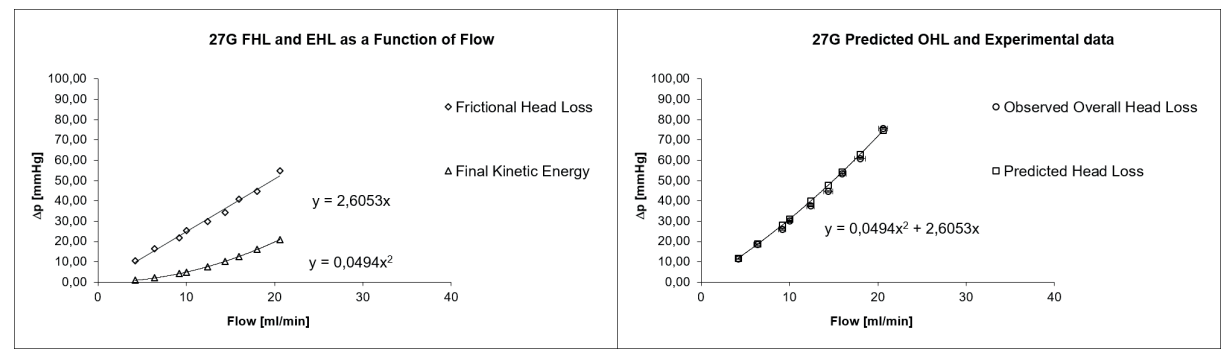

Fig. 7. $27 \mathrm{G}$ infusion. (Left) $\mathrm{EHL}$ and $\mathrm{FHL}$ as functions of observed volumetric flow. Both curves and derived equations (EHL, parabolic and FHL, linear) are reported in each graph. (Right) Measured OHL compared to predictions by the derived equation (reported in the plot). Experimental data points (observed $\mathrm{OHL}$ ) and calculated values (predicted head loss) overlap significantly. 


\subsection{Interpolation of experimental data points with best fit polynomial equation}

In order to test whether a simple best fitting algorithm of experimental flow rate data per se could yield satisfying OHL prediction, we used the Excel 2013 (Microsoft, USA) best fitting curve to yield a 2 nd order polynomial equation (hereafter referred to as the interpolated equation).

We then calculated $\mathrm{OHL}$ values by the interpolating curve and plotted them in Figure 8 for comparison with experimental (observed) data and OHL values predicted by the derived function.

\subsection{Statistical analysis}

The t-test was used for repeated measures of flow rate. Statistical significance was set at a $p$-value less than 0.05 .

The Bland-Altman statistic evaluated the agreement between observed and $\mathrm{OHL}$ values predicted with the derived and interpolated functions. According to Bland and Altman recommendations, ${ }^{8,9}$ the agreement was considered statistically significant if at least $95 \%$ of the data points fell within \pm 2 SD of the mean difference between observed and predicted OHL.

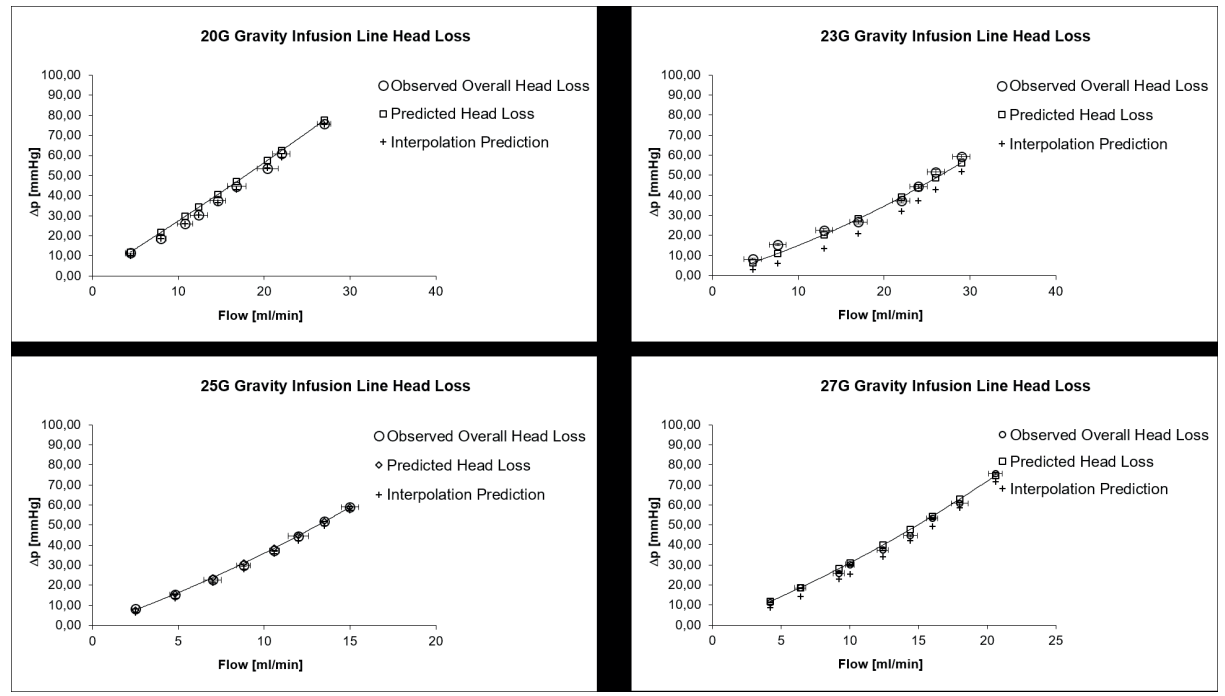

Fig. 8. Comparison of infusion line head loss: (Top left) 20G; (Top right) 23G; (Bottom left) 25G; (Bottom right) 27G. Each graph reports the comparison between experimental data points (observed), derived equation, and interpolated data prediction and curves. For each tested caliper, the derived equation yields predicted values closer to experimental ones (observed). 


\section{Results}

Figure 2 reports volumetric FR as a function of the DE for all tested gauges. FR decreased in the following order: $23 \mathrm{G}>20 \mathrm{G}>27 \mathrm{G}>25 \mathrm{G}$; the difference between all calipers reached statistical significance for any given pressure delta over $15 \mathrm{mmHg}$ $(p<0.01)$.

The EHL parabolic function and FHL linear equation fitting experimentally derived points are reported in the left panes of Figures 4-7, while the comparison between experimental points (observed) and the derived function (see Appendix) for all tested gauges are shown in the right panes of Figures 4-7. The graphs report the comparison between experimental data points (observed), derived equation, and interpolated data prediction and curves. For each tested caliper, the derived equation yields predicted values closer to experimental ones (observed). Figure 8 includes the 2nd order polynomial best fit in the comparison.

The Bland-Altman plot of observed OHL vs those predicted with both equations is shown in Figure 9. The derived function (Fig. 10a) shows a highly statistically significant agreement with experimental data: $97.1 \%$ of all data points falling within 2 SD of the mean difference. The best fit (interpolation) function (Fig. 10b) showed a higher SD and data scattering with only $91.1 \%$ of predicted values falling within \pm 2 SD of the mean difference, a predictive value considered non-significant.

The difference between $\mathrm{OHL}$ data calculated with the derived and interpolated functions proved also highly statistically significant $(p<0.01)$.
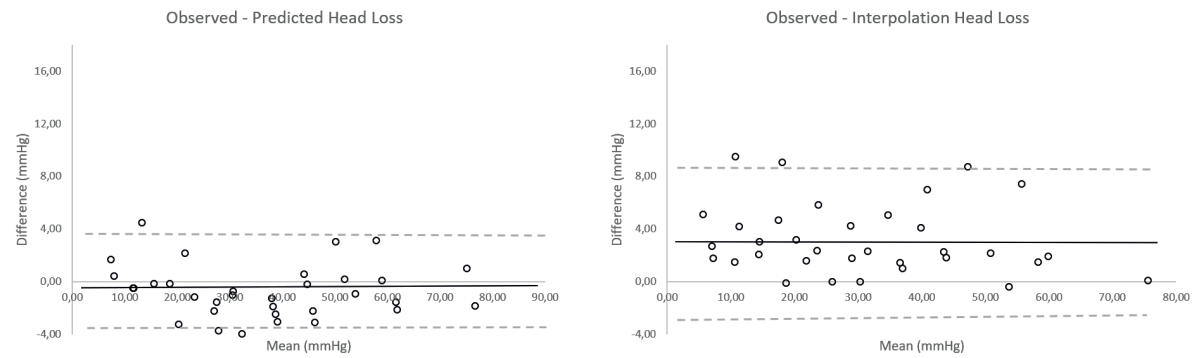

Fig. 9. Bland-Altman plot for (left) predicted and (right) interpolated values. Predicted values are less dispersed and much closer to the $x$-axis (97\% within 2 SD) than those obtained with the interpolated function (91\% within 2 SD). Solid line is the mean of difference and dotted line \pm 2 SD. 


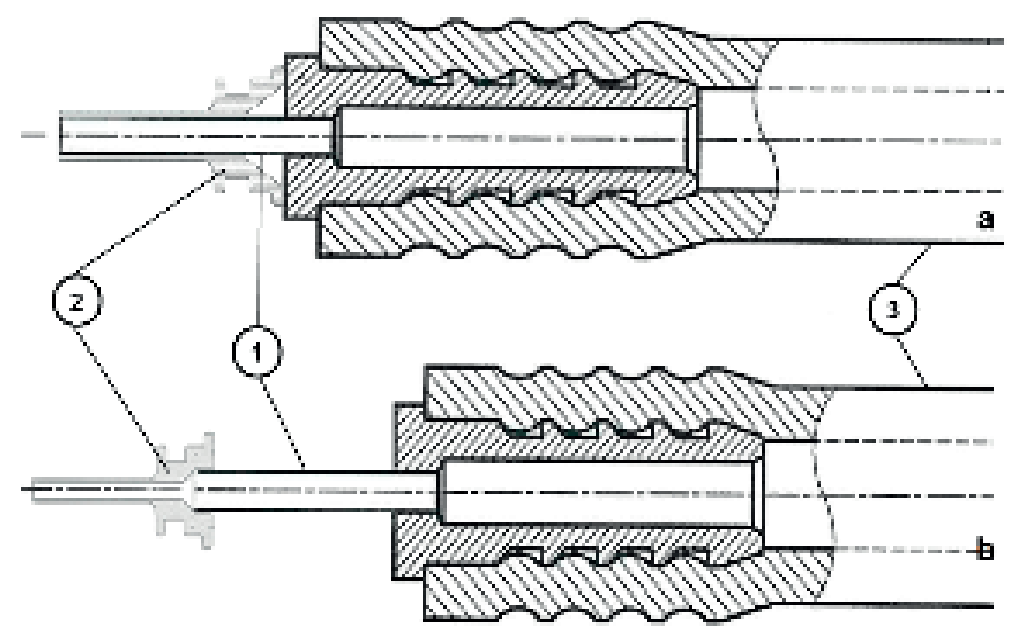

Fig. 10. Schematics of the trocar/cannula systems used during the experiments. (a) 20G, 23G, and 25G. (b) 27G. (1) infusion tubing metal connector; (2) trocar; (3) silicone tubing.

\section{Discussion}

Maintaining a stable IOP throughout surgery is extremely important, as pressure changes may result in severe intra- and postoperative complications, as described by Minami et al. ${ }^{10}$ Previous studies by Kim et al. ${ }^{11}$ and Moorhead et al..$^{12}$ on PPV recorded an IOP range of $0-120 \mathrm{mmHg}$, with oscillations exceeding $40 \mathrm{mmHg}$, values which may induce acute ischemic damage also in healthy eyes. ${ }^{13}$

The introduction of small gauge instruments with highly efficient pumps allows high outflow rates and reduced surgical time, but inherently raises the issue of head loss and the risk of hypotony, as clearly shown in the work of Abulon et al. ${ }^{14}$ In fact, small gauge surgery imposes a narrow distal infusion opening that limits inflow, increasing the risk of significant pressure drop, especially at high vacuum.

The purpose of our study is to characterize the flow of 20G through 27G infusion lines in order to measure head loss and define a consistent prediction algorithm as a prerequisite for its efficient compensation.

Experimental data (Fig. 2) emphasize the objective clinical significance of head loss and the need for $\mathrm{YOHL}$ compensation, showing head losses corresponding up to $40 \mathrm{mmHg}$ at flow easily generated during surgery. The relative role of tubing and trocar/cannula in $\mathrm{OHL}$ is clarified in the left panes of Figures 4-7: the tubing frictional component is predominant (steeper curve), but the trocar/cannula gains significance as flow increases and caliper diminishes, reaching a fraction as high as approximately one-third of the FHL (left panels in Figs. 5-7). It should be noted that 
FKE includes the energy dissipation along the tubing, predicted by the Hagen-Poiseuille law and the additional head loss due to the sudden variations, such as tips or connections between different conduit tracts.

Construction details explain the apparently odd result of the $27 \mathrm{G}$ and $23 \mathrm{G}$ yielding a lower EHL compared to the $25 \mathrm{G}$ and $20 \mathrm{G}$, respectively. Unlike their counterparts, the $27 \mathrm{G}$ and $23 \mathrm{G}$ infusion tubing are in fact directly connected to the trocar, whereas the $25 \mathrm{G}$ and $20 \mathrm{G}$ have a metal cannula tip sliding into the trocar, thus reducing its inner lumen (see Fig. 10 and Table 1). Additionally, the FHL is slightly lower for the $27 \mathrm{G}$ infusion compared to the $25 \mathrm{G}$. This demonstrates that a significant fraction of frictional dissipation (FHL) occurs at the narrowest section: the trocar/cannula system. The meaningful role that the trocar/cannula system plays both in EHL and FHL suggests that its design is crucial in minimizing the pressure drop generated by an infusion line. Interestingly, since head loss depends on the $4^{\text {th }}$ power of the lumen diameter based on Hagen-Poiseuille law for fluid flow, even a small increase of the exit lumen and/or inner diameter of the cannula inner lumen may significantly decrease the related head losses.

We derived head loss function (OHL) by adding its known physical components: EHL and FHL. The former has a known equation (see Methods section and Appendix), while the latter is obtained from experimental data (Figs. 4-7). The developed algorithms are both justified from a purely hydraulic standpoint and experimentally validated, since predicted $\mathrm{OHL}$ values closely matched experimental data (right panels in Figs. 4-7 and Fig. 8; 20G-27G) and proved significantly more accurate than direct interpolation (Figs. 8 and 9). It is important to note that the coefficients of the equation proposed here strictly refer to tested infusion lines and cannot be applied to infusion tubing belonging to other manufacturers. Nonetheless, their form holds in general and the same simple method can be used to derive the appropriate coefficients for any given infusion line of any brand.

We relied on gravity infusion to ensure invariant pressure given by piezometric head and yield accurate measures. Although most vitrectomy machines use a forced infusion system, the concept of head loss applies to pressure drop caused by flow, regardless of how pressure is imposed on the infusion bottle.

An accurate $\mathrm{OHL}$ compensating system would undoubtedly represent a step forward although insufficient per se in guaranteeing a stable pressure throughout surgery. A 'feed forward' mechanism, in fact, may correct deterministic, systematic biases due to the onset and interruption of aspiration, but are useless by definition in counteracting random pressure changes as well as transient states. Feedback controls, on the contrary, can compensate unpredictable changes but take time, and require fine-tuning to avoid dangerous overcorrection and/or system divergence.

Falabella et al. ${ }^{4}$ and Sugiura et al. ${ }^{15}$ investigated one such device and concluded that it reestablished preset IOP after an average delay of 2.8 seconds; a lengthy time and potentially dangerous, due to the system design that reacts ex-post to pressure drop. 
Falabella et al. ${ }^{4}$ also reported an overshooting pressure wave after head loss compensation, but did not investigate the opposite phenomenon. When high flow aspiration steady state is reached, the system increases infusion pressure to balance head loss; if aspiration stops abruptly, eye pressure will suddenly equal the infusion line pressure at potentially dangerously high levels. ${ }^{10}$ In such a case, flow would stop almost instantaneously and no compensating system could effectively attenuate this pressure rise.

In summary, we derived functions capable of describing with very high precision $\mathrm{OHL}$ based on flow in a steady regime. Although unable to capture the transient effects due to the velocity of variation of the FR, the equations based analytically on hydraulics laws demonstrated a statistically significant prediction capability of the steady component of the pressure variations. The same method can be used for any given infusion system and will help develop algorithms capable of improving intraoperative pressure control. It is worth noting that, in principle, the present study could be extended to investigate the transient pressure changes due to the flow variation velocity. However, these effects are presumably too fast to be reliably compensated in real time during surgery.

\section{Acknowledgements}

Giampiero Angelini, Alessandro Rossi, and Carlo Malvasi are employees of Optikon 2000 Inc. (Rome, Italy). None of the other authors has any financial interest in the subject matter and no financial support is involved in the study. The authors wish to thank the Fondazione Roma (Rome, Italy) for its support.

\section{References}

1. Rossi T, Querzoli G, Angelini G, Rossi A, Malvasi C, lossa M, Ripandelli G. Ocular perfusion pressure during pars plana vitrectomy: a pilot study. Invest Ophthalmol Vis Sci. 2012;55:8497-8505.

2. Rossi T, Querzoli G, Gelso A, Angelini G, Rossi A, Corazza P, Landi L, Telani S, Ripandelli G. Ocular perfusion pressure control during pars plana vitrectomy: testing a novel device. Graefes Arch Clin Exp Ophthalmol. 2017 Sep 8. doi: 10.1007/s00417-017-3799-2

3. Abulon DJ, Buboltz DC. Performance Comparison of High-Speed Dual-Pneumatic Vitrectomy Cutters during Simulated Vitrectomy with Balanced Salt Solution. Transl Vis Sci Technol. 2015;29:4-6.

4. Falabella P, Stefanini FR, Lue JC, et al. Intraocular pressure changes during vitrectomy using Constellation vision system's intraocular pressure control feature. Retina. 2016;36:1275-1280.

5. Okamoto F, Sugiura Y, Okamoto Y, Hasegawa Y, Hiraoka T, Oshika T. Measurement of ophthalmodynamometric pressure with the vented-gas forced-infusion system during pars plana vitrectomy. Invest Ophthalmol Vis Sci. 2010;51:4195-4199

6. Sutera, SP, Skalak, R. The History of Poiseuille's Law. Annu Rev Fluid Mech. 1993;25:1-20. https://doi. org/10.1146/annurev.fl.25.010193.000245 
7. Poiseuille, J. L. M. Recherches experimentales sur le mouvement des liquides dans les tubes de tres petits diametres; II. Influence de la longueur sur la quantite de liquide qui traverse les tubes de tres petits diametres; III. Influence du diametre sur la quantite de liquide qui traverse les tubes de tres petits diametres. C. R. Acad. Sci.1840; 11:1041-1048

8. Bland JM, Altman DG. Statistical methods for assessing agreement between two methods of clinical measurement. Lancet. 1986;2:307-310.

9. Bland JM, Altman DG. Comparing methods of measurement: why plotting difference against standard method is misleading. Lancet. 1995;346:1085-1087.

10. Minami M1, Oku H, Okuno T, Fukuhara M, Ikeda T. High infusion pressure in conjunction with vitreous surgery alters the morphology and function of the retina of rabbits. Acta Ophthalmol Scand. 2007;85:633-639.

11. Kim YJ, Park SH, Choi KS. Fluctuation of infusion pressure during microincision vitrectomy using the constellation vision system. Retina. 2015;5:2529-2536.

12. Moorhead LC, Gardner TW, Lambert HM, O'Malley RE, Willis AW, Meharg LS, Moorhead WD. Dynamic intraocular pressure measurements during vitrectomy. Arch Ophthalmol. 2005;123:1514-1523.

13. Michelson G, Groh MJ, Langhans M. Perfusion of the juxtapapillary retina and optic nerve head in acute ocular hypertension. Ger J Ophthalmol. 1996;5:315-321

14. Abulon DJ, Buboltz DC. Performance Comparison of High-Speed Dual-Pneumatic Vitrectomy Cutters during Simulated Vitrectomy with Balanced Salt Solution. Transl Vis Sci Technol. 2015;29:4-6.

15. Sugiura Y, Okamoto F, Okamoto Y, Hiraoka T, Oshika T. Intraocular pressure fluctuation during microincision vitrectomy with constellation vision system. Am J Ophthalmol. 2013;156:941-947. 


\section{Appendix}

\section{Derived function}

Figure 2a reports experimental $\Delta \mathrm{p}_{\mathrm{FHL}}$ and $\Delta \mathrm{p}_{\mathrm{EHL}}$ data in $\mathrm{mmHg}$ as functions of $\mathrm{FR}$ measured in $\mathrm{ml} / \mathrm{min}$ for the $20 \mathrm{G}$ infusion line. The $\mathrm{EHL}$ in $\mathrm{mmHg}$ was evaluated as a function of $\mathrm{FR}$ in $\mathrm{ml} / \mathrm{min}$ using Equation (3) that assumes the form:

$$
\Delta \mathrm{pFHL}=\mathrm{a} \times \mathrm{FR}^{2}
$$

where the coefficient $\mathrm{a}=0.0075 \mathrm{mmHg} /\left(\mathrm{ml}^{2} / \mathrm{min}^{2}\right)$.

The FHL computed by Equation (2) is then approximated by a linear best fit as a function of the $\mathrm{FR}$ with the equation:

$$
\Delta \mathrm{p}_{\mathrm{FHL}}=\mathrm{b} \times \mathrm{FR}
$$

where $b=2.6700 \mathrm{mmHg} /(\mathrm{ml} / \mathrm{min})$.

Since $\mathrm{OHL}=\mathrm{FKE}+\mathrm{FKL}$, the pressure drop, which equals $\mathrm{\gamma OHL}$, can be predicted as the sum of $\Delta \mathrm{p}_{\mathrm{EHL}}$ and $\Delta \mathrm{p}_{\mathrm{FHL}}$ equations as follows (derived from the OHL equation):

$$
\Delta p=\gamma O H L=a \times F R^{2}+b \times F R
$$

The left panel in Figure 2 reports $\Delta \mathrm{p}$ obtained from the measured $\mathrm{OHL}$ and the predicted head loss as a function of FR, calculated with the above equation for the $20 \mathrm{G}$ infusion line. The overlap between experimental and prediction data is excellent.

\section{Interpolation function}

For comparison, a 2nd-degree polynomial interpolant was obtained by a best fit procedure from $\mathrm{OHL}$ data points, viz:

$$
\Delta \mathrm{p}=\mathrm{\gamma OHL}=\mathrm{c} \times \mathrm{FR}^{2}+\mathrm{d} \times \mathrm{FR}
$$

where $\mathrm{c}=0.0242 \mathrm{mmHg} /\left(\mathrm{ml}^{2} / \mathrm{min}^{2}\right)$ and $\mathrm{d}=2.1463 \mathrm{mmHg} /(\mathrm{ml} / \mathrm{min})$; the pressure drop is measured in $\mathrm{mmHg}$ and $\mathrm{FR}$ in $\mathrm{ml} / \mathrm{min}$, as usual. The values for the pressure drop obtained from the interpolating Equation (7), from the derived Equation (6), and the experimental data are plotted in the left panel of Figure 6. 
The same procedure was repeated for the 23G, 25G, and 27G infusion circuits. Corresponding coefficients $\mathrm{a}$ and $\mathrm{b}$ of the derived Equation (6) and $\mathrm{c}$ and $\mathrm{d}$ of the interpolant Equation (7) are listed in Table 1 and related data is plotted in Figures 5-8. 